\section{Estratégia Saúde da Família em comparação a outras fontes de atenção: indicadores de uso e qualidade dos serviços de saúde em Belo Horizonte, Minas Gerais, Brasil}

\author{
A comparison of the Family Health Strategy to \\ other sources of healthcare: utilization and quality \\ of health services in Belo Horizonte, Minas Gerais \\ State, Brazil
}

\section{Estrategia Salud de la Familia en comparación con otras fuentes de atención: indicadores de uso y calidad de los servicios de salud en Belo Horizonte, Minas Gerais, Brasil}

Maria Fernanda Lima-Costa 1,2 Maria Aparecida Turci 2 James Macinko ${ }^{3}$

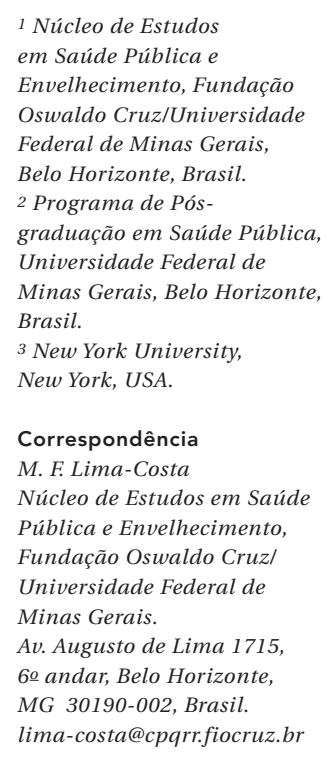

1 Núcleo de Estudos em Saúde Pública e Envelhecimento, Fundação Oswaldo Cruz/Universidade Federal de Minas Gerais, Belo Horizonte, Brasil. 2 Programa de Pósgraduação em Saúde Pública, Universidade Federal de Minas Gerais, Belo Horizonte, Brasil.

${ }^{3}$ New York University, New York, USA

Correspondência M. F. Lima-Costa Núcleo de Estudos em Saúde Pública e Envelhecimento, Fundação Oswaldo Cruz/ Universidade Federal de Minas Gerais. Av. Augusto de Lima 1715, 60 andar, Belo Horizonte, MG 30190-002, Brasil. lima-costa@cpqrr.fiocruz.br

\begin{abstract}
Indicators of healthcare utilization and quality were compared in a probabilistic sample of adults $(N=7,534)$ covered by private health plans, the Family Health Strategy (FHS), and "traditional" primary care clinics (UBS) in Belo Horizonte, Minas Gerais State, Brazil. After adjusting for demographics, health conditions, and socioeconomic status, indicators of healthcare utilization (longitudinality, health-seeking, and medical consultations) showed better performance among users of the FHS and private health plans compared to those covered by the UBS. Hospitalizations, preventive tests, and flu vaccinations varied little between sources of care. Quality indicators (difficulty in making an appointment, waiting lines, complaints about obtaining medications, and receiving an appointment within 24 hours) were better among private health plans. Recommending one's healthcare providers to others was more frequent among FHS users (61.9\%) and those with private health plans (55.6\%), compared to those served by UBS (45.4\%).
\end{abstract}

Health Services Accessibility; Health Care Quality Indicators; Prepaid Health Plans

\section{Resumo}

Foram comparados indicadores de uso e qualidade dos serviços de saúde em uma amostra probabilística de adultos ( $N=7.534)$ cobertos por plano privado, Estratégia Saúde da Família (ESF) e unidade básica de saúde (UBS) "tradicional" no Município de Belo Horizonte, Minas Gerais, Brasil. Após ajustamentos por fatores demográficos, condições de saúde e situação socioeconômica, os indicadores de uso (longitudinalidade, procura por atenção e consultas médicas) apresentaram melhor performance entre usuários regulares da ESF e afiliados a plano privado em comparação aos cobertos pela UBS. As hospitalizações, os exames de rastreamento e a vacinação contra a gripe variaram pouco entre esses grupos. Os indicadores de qualidade (dificuldades para obter consultas, existência de filas, queixa para obtenção de medicamentos e obtenção de consultas em 24 horas) foram melhores entre afiliados a plano privado. A recomendação para outra pessoa dos serviços de saúde utilizados foi mais frequente entre usuários regulares da ESF (61,9\%) e afiliados a plano privado (55,6\%), em comparação à UBS $(45,4 \%)$.

Acesso aos Serviços de Saúde; Indicadores de Qualidade em Assistência à Saúde; Planos de Pré-pagamento em Saúde 


\section{Introdução}

A atenção primária é baseada em um modelo, visando ao cuidado longitudinal do indivíduo para a maioria dos problemas e necessidades em saúde, a atenção integral, a provisão de cuidados no contexto da família e da comunidade, assim como a coordenação dos diferentes níveis de atenção ${ }^{1}$. A atenção primária, por meio da Estratégia Saúde da Família (ESF), é uma das prioridades do Sistema Único de Saúde (SUS), com crescente comprometimento orçamentário do Ministério da Saúde para a mesma 2,3.

A avaliação da ESF, por meio de estudos epidemiológicos, vem recebendo atenção crescente. Estudos ecológicos, baseados em dados secundários de abrangência nacional, mostraram que a expansão da ESF está associada a reduções da mortalidade infantil 4,5 e de hospitalizações por condições sensíveis à atenção primária (ou seja, internações evitáveis por ações adequadas nesse nível de atenção) 6,7,8. Um estudo transversal, baseado em dados da Pesquisa Nacional por Amostra de Domicílios (PNAD 2008) e utilizando o método de pareamento pelo escore de propensão, mostrou que adultos cobertos pela ESF eram mais propensos a ter uma fonte regular de cuidados com a saúde, a obter consultas médicas e odontológicas, a ter acesso a medicamentos de uso regular e a estar satisfeitos com a atenção à saúde recebida, em comparação aos não cobertos pela ESF ou afiliados a plano privado de saúde. Essas associações foram mais fortes entre aqueles com pior situação socioeconômica 9. Outros estudos epidemiológicos transversais, conduzidos em municípios com mais de 100 mil habitantes nas regiões Sul e Nordeste do Brasil, mostraram que adultos portadores de doenças crônicas e cobertos pela ESF realizavam mais consultas médicas que seus correspondentes cobertos pelo modelo tradicional de atenção primária ${ }^{10}$. Além disso, em comparação aos últimos, os cobertos pela ESF na Região Nordeste (mas não na Região Sul) tinham mais acesso a medicamento de uso regular 11 .

Apesar da alta cobertura, alguns autores lembram que existem potenciais limitações para a consolidação da ESF como organizador da atenção primária em grandes centros urbanos, sobretudo por causa da diversidade de outros modelos de atenção 12. Entretanto, pouco se sabe acerca da performance da ESF em capitais brasileiras. No Município de São Paulo, verificou-se que um índice de atenção primária (constituído por diferentes dimensões desse modelo de atenção) era mais alto entre usuários das unidades da ESF, em comparação aos usuários das unidades básicas de saúde "tradicionais" 13. No Município de Belo
Horizonte, Minas Gerais, resultados de um estudo ecológico mostraram que havia uma correlação entre expansão da ESF e diminuição das hospitalizações por condições sensíveis à atenção primária ${ }^{14}$.

O presente estudo epidemiológico de base populacional, conduzido no Município de Belo Horizonte, tem por objetivo comparar indicadores de uso e qualidade dos serviços de saúde entre residentes em área coberta pela ESF, residentes em área coberta pela unidade básica de saúde (UBS) "tradicional" e afiliados a plano privado de saúde.

\section{Métodos}

\section{Área do estudo}

Belo Horizonte, capital do Estado de Minas Gerais, é uma das maiores cidades brasileiras, com 2,4 milhões de habitantes, ocupando uma área de $331 \mathrm{~km}^{2} 15$. Embora apresente o quarto maior Produto Interno Bruto do país e alto Índice de Desenvolvimento Humano $(0,839)$, existem profundas desigualdades sociais e das condições de saúde entre seus habitantes 16,17. Visando ao planejamento das ações de saúde, a Secretaria Municipal de Saúde de Belo Horizonte (SMS$\mathrm{BH})$ desenvolveu um índice de vulnerabilidade à saúde (IVS). Esse índice é baseado nas características dos moradores de cada setor censitário, compreendendo condições de saneamento, características do domicílio, escolaridade e renda dos indivíduos e indicadores da mortalidade precoce 16,18. Com base nesses componentes, os setores censitários são classificados em risco baixo, médio, elevado ou muito elevado. As áreas de risco médio, elevado e muito elevado foram selecionadas para a implantação da ESF a partir de 2003 18. Entre 2003 e 2010, o número de equipes da ESF aumentou de 473 para 504, alcançando a cobertura de $71 \%$ do total da população adulta no ano final (Departamento de Atenção Básica, Secretaria de Assistência à Saúde, Ministério da Saúde. Evolução do credenciamento e implantação da Estratégia Saúde da Família. http://dab. saude.gov.br/historico_cobertura_sf.php, acessado em 04/Mar/2012). Ao longo desse período, todas as UBS "tradicionais" nas áreas de cobertura da ESF foram substituídas pelo novo modelo de atenção, ao passo que nas áreas de risco baixo, a modalidade "tradicional" de atenção à saúde permaneceu. 


\section{População estudada e coleta de dados}

Foram utilizados dados do segundo Inquérito de Saúde da Região Metropolitana de Belo Horizonte, conduzido entre maio e julho de 2010. A coleta de dados foi realizada por meio de um questionário suplementar à Pesquisa de Emprego e Desemprego na Região Metropolitana de Belo Horizonte (PED/RMBH), conduzida pela Fundação João Pinheiro, órgão do governo do Estado de Minas Gerais 19. A PED/RMBH é realizada periodicamente, em uma grande amostra, baseada em 7.500 domicílios com cerca de 24 mil moradores. A amostra da PED foi delineada para produzir estimativas da população não institucionalizada com idade igual ou superior a dez anos residente em 26 municípios da Região Metropolitana de Belo Horizonte. Trata-se de uma amostra probabilística por conglomerados, estratificada em dois estágios. Os setores censitários do Instituto Brasileiro de Geografia e Estatística (IBGE) foram usados como unidade primária de seleção e a unidade amostral foi o domicílio. Para o cálculo amostral, estimou-se uma não resposta de $20 \%$ dos domicílios 19.

Do total de domicílios amostrados na Região Metropolitana de Belo Horizonte, 5.798 (77,3\%) participaram da pesquisa. Todos os residentes nos domicílios amostrados, com idade igual ou superior a 20 anos, foram elegíveis para a entrevista face a face do inquérito de saúde. Do total de 12.979 entrevistados na faixa etária elegível, 7.778 eram residentes no Município de Belo Horizonte e foram selecionados para a presente análise. Maiores detalhes podem ser vistos em outras publicações 17,19.

O segundo Inquérito de Saúde da Região Metropolitana de Belo Horizonte foi aprovado pelo Comitê de Ética do Instituto de Pesquisas René Rachou, da Fundação Oswaldo Cruz (carta de aprovação no 10/2009).

\section{Fontes de atenção à saúde}

A variável fontes de atenção à saúde foi construída com base na localização dos domicílios (se em área coberta ou não coberta pela ESF) e na informação individual de ser afiliado a plano privado de saúde. Todos os domicílios participantes da pesquisa foram georreferenciados para possibilitar a sua localização em cada setor censitário. Com base nessa localização, os participantes do estudo foram classificados em cobertos ou não pela ESF, considerando-se os parâmetros estabelecidos pela SMS-BH 18 .

Assim, a população do estudo foi classificada nos seguintes grupos, que são mutuamente excludentes: (1) cobertos pela UBS "tradicional" (não possui plano privado de saúde e reside em área não coberta pela ESF) (grupo I); (2) possui plano privado de saúde, independentemente do local de residência (grupo II); (3) residente em área coberta pela ESF e usuário não regular da mesma (nega ou não sabe informar se é atendido ou possui vizinhos atendidos pela ESF, além de não possuir plano privado de saúde) (grupo III); e (4) residente em área coberta pela ESF e provável usuário regular da mesma (afirma ser atendido ou possuir vizinho atendido pela ESF e não possui plano privado de saúde) (grupo IV).

\section{Indicadores de uso e qualidade dos serviços de saúde}

Os indicadores de uso de serviços de saúde incluíram: fonte regular de serviços de saúde (definido pelo relato de ter um determinado serviço de saúde ou médico que costuma procurar quando necessita de cuidados com a saúde); procura por atenção à saúde nas últimas duas semanas; realização de uma ou mais consultas médicas nos últimos 12 meses e ocorrência de uma ou mais hospitalizações (exceto parto) nos últimos 12 meses.

Os indicadores de qualidade incluíram qualidade da atenção na percepção do usuário, exames de rastreamento e vacinação contra a gripe. A qualidade dos serviços de saúde na percepção dos usuários foi avaliada por intermédio de cinco indicadores específicos e um indicador geral. Os indicadores específicos incluíram: relato de alguma dificuldade para conseguir consultas médicas quando necessita (dificuldades de acesso ao local, existência de filas, ausência do médico no local, preço da consulta e outras razões); existência de filas como principal dificuldade para conseguir consultas médicas entre aqueles que informaram ter alguma dificuldade; relato de alguma dificuldade para obter medicamentos entre aqueles que fazem uso regular dos mesmos (dificuldades devido ao preço, falta do medicamento na farmácia ou no local de distribuição, acesso à farmácia ou local de distribuição e outras razões); frequência com que consegue consultas médicas num prazo de 24 horas no serviço ou médico que costuma procurar; frequência com que considera que o atendimento dura o tempo necessário no serviço ou médico que costuma procurar. Foi considerada indicador global de satisfação a recomendação para outras pessoas da maioria dos serviços de saúde ou dos médicos que costuma procurar.

Entre os exames de rastreamento, a aferição da pressão arterial, a realização do exame do Papanicolau e a realização da mamografia, nos intervalos e nas faixas etárias recomendadas pelo 
Ministério da Saúde 20,21, foram incluídas na presente análise, tendo 20 anos de idade como limite inferior em função do critério de elegibilidade para o inquérito de saúde. Assim, foram consideradas a realização de uma ou mais medidas da pressão arterial nos últimos dois anos entre homens e mulheres com idade igual ou superior a 20 anos, realização da mamografia nos últimos dois anos entre mulheres com 50-69 anos, realização do exame do Papanicolau nos últimos três anos em mulheres com 25-59 anos que possuem útero. Embora a recomendação do Ministério da Saúde seja a aferição do colesterol entre homens e mulheres com 35 e 45 anos ou mais de idade, respectivamente, optamos por utilizar a recomendação norte-americana para a realização desse exame em toda a população adulta 22; tal opção foi feita em função da altíssima cobertura do exame de colesterol na população estudada. Finalmente, foi considerada a vacinação contra a gripe nos últimos 12 meses entre homens e mulheres com 60 anos ou mais de idade.

\section{Variáveis de confusão}

O modelo teórico para a seleção de variáveis de confusão foi adaptado daquele proposto por Andersen et al. 23 , tendo em conta fatores predisponentes, de necessidade (condições de saúde) e facilitadores do uso de serviços de saúde. Entre os fatores predisponentes, foram incluídos idade, sexo e presença de cônjuge. Entre os fatores de necessidade foram considerados autoavaliação da saúde (baseado na resposta à pergunta "De maneira geral o senhor diria que sua saúde é?", com respostas variando entre muito boa a muito ruim), interrupção das atividades rotineiras nos últimos trinta dias em razão de problemas de saúde, número de dias nos quais a saúde física não foi boa nos últimos trinta dias; número de dias nos quais a saúde mental não foi boa nos últimos trinta dias e história de diagnóstico médico para doenças crônicas ou condições crônicas (baseada na pergunta "Algum médico já disse que você tem...”). As doenças e condições crônicas consideradas foram hipertensão, diabetes e bronquite/asma, infarto do miocárdio ou angina do peito, outra doença do coração, acidente vascular cerebral, câncer, doença renal crônica e dores crônicas da coluna. Entre os fatores facilitadores, foram considerados a escolaridade do indivíduo e os bens domiciliares (número de aparelhos de televisão em cores, rádios, banheiros, automóveis, máquinas de lavar roupas, aparelhos de videocassetes ou de DVD, geladeiras, freezers, telefones fixos, telefones celulares e computadores), além da existência de um ou mais empregados domésticos.

\section{Análise estatística}

As análises univariadas foram baseadas no teste do qui-quadrado de Pearson e na regressão linear para examinar a significância estatística de diferenças entre frequências e proporções, respectivamente. As análises multivariadas foram baseadas em duas etapas. Primeiro, foi implementada a regressão de Poisson com variância robusta para eventos comuns 24,25 para examinar a significância estatística das associações entre a fonte de cuidados com a saúde e os indicadores de uso e qualidade dos serviços de saúde, considerando-se o grupo I (UBS "tradicional") como referência. Por essas estimativas, foram calculadas prevalências ajustadas e intervalos de $95 \%$ de confiança (IC95\%). Todas as análises multivariadas foram ajustadas por faixa etária (20-39, 40-59 e 60 anos ou mais), sexo, presença de cônjuge (sim, não), escore de condições de saúde, escore de bens domiciliares e nível de escolaridade (até 3, 4-7 e 8 ou mais anos completos). Por conta da existência de correlação interna, foram criadas duas variáveis latentes, mediante análise de componentes principais 26 . Para a construção do escore de condições de saúde, foram considerados os seguintes indicadores: autoavaliação da saúde como ruim/muito ruim (vs. melhor), número de dias nos quais a saúde física e a saúde mental não foram boas (ambas como variáveis contínuas), e história de diagnóstico médico para uma ou mais doenças crônicas (uma variável dicotômica). Para a construção do escore de bens domiciliares, foram considerados todos os bens do domicílio (cada um como variável contínua) e a existência de empregados domésticos (variável dicotômica). Após distribuição de cada escore, o de saúde foi dividido em tercis e o de condições socioeconômicas em quintis.

As análises foram conduzidas com uso da versão 11.0 do pacote estatístico Stata (Stata Corp., College Station, Estados Unidos), incorporandose os pesos individuais, o delineamento complexo da amostra e o efeito da agregação domiciliar.

\section{Resultados}

Dos 7.778 participantes do inquérito de saúde residentes no Município de Belo Horizonte, $7.534(96,7 \%)$ possuíam informações sobre as variáveis de interesse do estudo e foram incluídos nesta análise. No total, 1.014 (13,5\%) foram classificados no grupo I (cobertos pela UBS "tradicional”), 3.519 (46,7\%) no grupo II (cobertos por plano privado de saúde), 1.111 (14,8\%) no grupo III (cobertos pela ESF e não usuários regulares dela) e $1.890(25,1 \%)$ no grupo IV (cober- 
tos pela ESF e prováveis usuários regulares dela) (Tabela 1).

A média da idade, assim como o escore de condições de saúde, não diferiram significativamente entre os grupos acima mencionados, e a distribuição por sexo apresentou uma discreta variação entre eles. Por outro lado, o nível de escolaridade e o escore de bens domiciliares diferiram significativamente entre os grupos, com melhor situação entre afiliados a plano privado de saúde, seguidos pelos residentes em área coberta pela UBS "tradicional" e os residentes em área coberta pela ESF (grupos III e IV) (Tabela 1).

Na Tabela 2, estão apresentadas as distribuições dos indicadores de uso de serviços, segundo a fonte de atenção à saúde. A proporção dos que informaram ter uma fonte regular de serviços de saúde foi significativamente $(\mathrm{p}<0,05)$ mais alta entre os que possuíam plano privado de saúde (65,8\%) (grupo II) e entre os que residiam em área coberta pela ESF e eram prováveis usuários regulares dela (57\%) (grupo IV), em comparação aos residentes em área coberta pela UBS "tradicional” (48,5\%) (grupo I); a proporção correspondente foi mais baixa entre os residentes em área coberta pela ESF e usuários não regulares da mesma $(40,7 \%)$, independentemente de outros fatores relevantes. A proporção dos que procuraram por atenção em saúde nas últimas duas semanas foi maior nos grupos II e IV (13,2\% e $12,6 \%$, respec-

Tabela 1

Distribuição das características sociodemográficas e indicadores de condições de saúde segundo a fonte de atenção à saúde. Belo Horizonte, Minas Gerais, Brasil, 2010

\begin{tabular}{|c|c|c|c|c|}
\hline Características & $\begin{array}{l}\text { Grupo I: UBS } \\
\text { "tradicional" } \\
(n=1.014)\end{array}$ & $\begin{array}{l}\text { Grupo II: plano } \\
\text { privado de saúde } \\
\quad(n=3.519)\end{array}$ & $\begin{array}{l}\text { Grupo III: coberto } \\
\text { pela ESF e usuário } \\
\text { não regular } \\
(n=1.111)\end{array}$ & $\begin{array}{l}\text { Grupo IV: cobertos } \\
\text { pela ESF e provável } \\
\text { usuário regular } \\
(n=1.890)\end{array}$ \\
\hline Média da idade (DP) & $42,7(0,7)$ & $45,0(0,4)$ & $41,7(0,6)$ & $43,7(0,4)$ \\
\hline Sexo feminino * & 51,5 & 55,9 & 53,2 & 54,0 \\
\hline Possui cônjuge * & 44,6 & 54,0 & 49,6 & 51,9 \\
\hline Escolaridade até 1ㅇgrau * & 43,4 & 20,0 & 53,0 & 56,1 \\
\hline \multicolumn{5}{|l|}{ Bens do domicílio } \\
\hline$\geq 2$ televisões em cores * & 62,6 & 74,4 & 47,6 & 63,9 \\
\hline$\geq 2$ rádios * & 31,5 & 43,1 & 23,4 & 34,2 \\
\hline$\geq 2$ banheiros * & 43,8 & 60,5 & 26,2 & 44,1 \\
\hline$\geq 1$ automóvel * & 49,8 & 74,5 & 39,8 & 41,0 \\
\hline$\geq 1$ máquina lavar roupas * & 82,4 & 91,5 & 71,8 & 83,1 \\
\hline$\geq 2$ videocassetes ou DVD * & 14,3 & 24,7 & 16,7 & 8,1 \\
\hline$\geq 1$ geladeira * & 97,0 & 95,4 & 97,5 & 94,6 \\
\hline$\geq 1$ freezer $*$ & 42,3 & 55,6 & 29,4 & 45,1 \\
\hline$\geq 1$ telefone fixo * & 80,1 & 89,6 & 73,8 & 82,3 \\
\hline$\geq 2$ telefones celulares * & 73,4 & 81,5 & 70,9 & 76,4 \\
\hline$\geq 1$ computador * & 67,4 & 82,9 & 58,9 & 52,1 \\
\hline Possui empregado doméstico & 10,9 & 22,2 & 3,8 & 3,5 \\
\hline Quintil mais alto de bens domiciliares (mais ricos) & 23,1 & 42,3 & 13,6 & 9,4 \\
\hline Autoavaliação da saúde ruim ou muito ruim * & 3,7 & 2,6 & 3,7 & 4,4 \\
\hline \multicolumn{4}{|l|}{ nos últimos 30 dias } & 5,8 \\
\hline Saúde física ruim $\geq 3$ dias nos últimos 30 dias & 8,9 & 10,0 & 8,8 & 10,0 \\
\hline Saúde mental ruim $\geq 3$ dias nos últimos 30 dias & 3,0 & 2,8 & 1,5 & 2,6 \\
\hline Uma ou mais doenças crônicas & 32,9 & 34,7 & 30,1 & 34,3 \\
\hline Tercil mais alto de condições de saúde (pior) & 11,7 & 12,3 & 10,9 & 12,2 \\
\hline
\end{tabular}

DP: desvio-padrão; ESF: Estratégia Saúde da Família; UBS: unidade básica de saúde.

Nota: os resultados estão apresentados em porcentagem, exceto quando especificado.

${ }^{*} p<0,05$ (para diferenças entre os grupos). 
Indicadores de uso de serviços de saúde, segundo a fonte de atenção à saúde. Belo Horizonte, Minas Gerais, Brasil, 2010.

\begin{tabular}{|c|c|c|c|c|}
\hline Modelo de atenção & $\begin{array}{l}\text { Possui fonte regular de } \\
\text { serviços de saúde }\end{array}$ & $\begin{array}{l}\text { Procurou por atenção } \\
\text { à saúde nas } 2 \text { últimas } \\
\text { semanas }\end{array}$ & $\begin{array}{l}\text { Realizou uma ou mais } \\
\text { consultas médicas nos } \\
\text { últimos } 12 \text { meses }\end{array}$ & $\begin{array}{c}\text { Foi hospitalizado uma } \\
\text { ou mais vezes nos } \\
\text { últimos } 12 \text { meses }\end{array}$ \\
\hline & $\%$ (IC95\%) & \% (IC95\%) & $\%$ (IC95\%) & $\%$ (IC95\%) \\
\hline Grupo I: UBS "tradicional" & $48,5(44,1-52,9)$ & $10,7(8,9-12,5)$ & $56,7(52,9-60,5)$ & $3,1(2,0-4,1)$ \\
\hline Grupo II: plano privado de saúde & $65,8(63,3-68,2)$ * & $13,2(11,8-14,6)$ * & $73,4(71,3-75,4)$ * & $4,1(3,3-4,9)$ \\
\hline $\begin{array}{l}\text { Grupo III: coberto pela ESF e } \\
\text { usuário não regular }\end{array}$ & $40,7(36,2-45,3)$ * & $9,0(7,0-10,6)$ & $47,4(43,3-51,4)$ * & $1,9(1,2-2,7)$ \\
\hline $\begin{array}{l}\text { Grupo IV: cobertos pela ESF e } \\
\text { provável usuário regular }\end{array}$ & $57,0(53,4-60,7)$ * & $12,6(11,0-14,2)$ * & $62,4(59,6-65,3)$ * & $3,6(2,8-4,4)$ \\
\hline
\end{tabular}

ESF: Estratégia Saúde da Família; IC95\%: intervalo de 95\% de confiança; UBS: unidade básica de saúde.

Nota: porcentagem ajustada por fatores predisponentes, de necessidade e facilitadores do uso de serviços de saúde.

${ }^{*} p<0,05$ para diferenças em relação ao grupo I.

tivamente) e menor no grupo III (9\%), em comparação ao grupo I (10,7\%). A proporção dos que haviam tido uma ou mais consultas médicas nos últimos 12 meses também foi mais alta nos grupos II $(73,4 \%)$ e IV $(62,4 \%)$, em comparação ao grupo I $(56,7 \%)$ e mais baixa no grupo III $(47,4 \%)$. A ocorrência de uma ou mais hospitalizações nos últimos 12 meses não apresentou variação estatisticamente significante entre os quatro grupos.

Na Tabela 3, estão apresentadas as distribuições de indicadores de qualidade dos serviços de saúde na percepção do usuário, segundo o modelo de atenção. O relato de ter alguma dificuldade para conseguir consultas médicas foi duas vezes menos frequente entre os que possuíam plano privado de saúde (32,7\%), em relação aos demais grupos $(59,3 \%, 63,5 \%$ e $62,4 \%$ nos grupos I, III e IV, respectivamente). A existência de filas como principal dificuldade para conseguir consultas médicas, entre os que relataram alguma dificuldade para obtê-las, foi informada por $12,2 \%$ dos afiliados a plano privado de saúde e por entre $28,8 \%$ e $38,8 \%$ nos demais grupos. Em comparação aos residentes em área coberta pela UBS “tradicional” (23\%), a prevalência de dificuldades para a obtenção de medicamentos de uso regular foi mais baixa entre os afiliados a plano privado de saúde $(14,4 \%)$ e entre os que residiam em área coberta pela ESF (15,6\% entre usuários regulares e 16,7\% entre usuários não regulares). A obtenção de consultas no prazo de 24 horas foi informada por $83,1 \%$ dos afiliados a plano privado de saúde e por entre $63,2 \%$ e $68,4 \%$ dos demais grupos. A proporção dos que consideravam que a sua consulta dura o tempo necessário foi baixa em todos os grupos considerados, sem di- ferenças estatisticamente significantes entre eles (39,3\%, 39,6\%, 41,4\% e 35,8\% nos grupos I, II, III e IV, respectivamente). Em comparação aos residentes em área coberta pela UBS "tradicional", a proporção dos que recomendariam para outras pessoas a maioria dos serviços que costumam procurar foi significativamente mais alta nos grupos II e IV (55,6\% e 61,9\%, respectivamente), e mais baixa no grupo III $(37,7 \%)$, em comparação ao grupo I $(45,4 \%)$.

Na Tabela 4, observa-se que as prevalências da aferição da pressão arterial e do exame do Papanicolau foram mais altas no grupo II $(96,2 \%$ e $91,1 \%$, respectivamente), em comparação ao grupo I (89,9\% e 85,3\%, respectivamente). Em relação ao último (94,1\%), a prevalência da aferição colesterol foi mais alta no grupo II $(97,2 \%)$ e mais baixa no grupo III $(89,7 \%)$. As prevalências da realização da mamografia (variação entre 79,8\% e $85 \%$ ) e da vacinação contra a gripe (variação entre $78,9 \%$ e $84,8 \%$ ) não diferiram significativamente entre os grupos I, II, III, IV.

\section{Discussão}

Os resultados deste trabalho, baseado em uma grande amostra de base populacional, mostram que, em geral, os indicadores de usos de serviços de saúde apresentaram melhor performance entre os prováveis usuários regulares da ESF, assim como entre os afiliados a plano privado de saúde. Os indicadores de qualidade dos serviços de saúde na percepção dos usuários foram predominantemente melhores entre os afiliados a plano privado de saúde. Por outro lado, as coberturas 
Tabela 3

Indicadores de qualidade dos serviços de saúde na percepção dos usuários, segundo a fonte de atenção à saúde. Belo Horizonte, Mina Gerais, Brasil, 2010.

\begin{tabular}{|c|c|c|c|c|c|c|}
\hline Modelo de atenção & $\begin{array}{l}\text { Tem alguma } \\
\text { dificuldade } \\
\text { para conseguir } \\
\text { consultas } \\
\text { médicas quando } \\
\text { necessita }\end{array}$ & $\begin{array}{c}\text { A existência } \\
\text { de filas é a } \\
\text { principal queixa } \\
\text { para conseguir } \\
\text { consultas médica } \\
\text { entre os que } \\
\text { relatam alguma } \\
\text { dificuldade para } \\
\text { obtê-las }\end{array}$ & $\begin{array}{l}\text { Tem alguma } \\
\text { queixa quando } \\
\text { precisa de } \\
\text { medicamentos de } \\
\text { uso regular }\end{array}$ & $\begin{array}{l}\text { Sempre ou na } \\
\text { maioria das } \\
\text { vezes consegue } \\
\text { consulta no prazo } \\
\text { de } 24 \text { horas }\end{array}$ & $\begin{array}{c}\text { Sempre ou na } \\
\text { maioria das } \\
\text { vezes o seu } \\
\text { atendimento } \\
\text { dura o tempo } \\
\text { necessário }\end{array}$ & $\begin{array}{l}\text { Recomendaria } \\
\text { todos ou a } \\
\text { maioria desses } \\
\text { serviços (ou } \\
\text { médicos) para } \\
\text { outras pessoas }\end{array}$ \\
\hline & $\%$ (IC95\%) & $\%$ (IC95\%) & $\%$ (IC95\%) & $\%$ (IC95\%) & $\%$ (IC95\%) & $\%$ (IC95\%) \\
\hline Grupo I: UBS "tradicional" & $59,3(55,3-63,4)$ & $28,8(25,1-32,6)$ & $23,0(16,2-29,8)$ & $63,2(58,2-68,2)$ & $39,3(34,4-44,2)$ & $45,4(40,5-50,2)$ \\
\hline $\begin{array}{l}\text { Grupo II: plano privado de } \\
\text { saúde }\end{array}$ & $32,7(30,4-35,0)$ * & $12,2(10,5-13,9)$ * & $14,4(11,4-17,3)$ * & $83,1(81,1-85,0)$ * & $39,6(34,4-44,2)$ & $55,6(53,1-58,1)$ * \\
\hline $\begin{array}{l}\text { Grupo III: coberto pela ESF } \\
\text { e usuário não regular }\end{array}$ & $63,5(59,1-67,9)$ & $38,8(34,5-43,1)$ * & $16,7(12,5-21,0)$ * & $64,5(59,4-69,6)$ & $41,4(36,4-46,4)$ & $37,7(32,2-43,2)$ * \\
\hline $\begin{array}{l}\text { Grupo IV: cobertos pela ESF } \\
\text { e provável usuário regular }\end{array}$ & $62,4(58,8-66,0)$ & $35,3(31,7-39,0)$ * & $15,6(12,0-19,1)$ * & $68,4(65,0-71,8)$ & $35,8(32,3-39,4)$ & $61,9(57,9-66,0)$ * \\
\hline
\end{tabular}

ESF: Estratégia Saúde da Família; IC95\%: intervalo de 95\% de confiança; UBS: unidade básica de saúde.

Nota: porcentagem ajustada por fatores predisponentes, de necessidade e facilitadores do uso de serviços de saúde.

* $p<0,05$ para diferenças em relação ao grupo 1 .

Tabela 4

Realização de exames preventivos e vacinação contra a gripe, segundo a fonte de atenção à saúde. Belo Horizonte, Minas Gerais, Brasil, 2010.

\begin{tabular}{|c|c|c|c|c|c|}
\hline \multirow[t]{3}{*}{ Modelo de atenção } & \multicolumn{2}{|c|}{ Homens e mulheres com 20 anos ou mais } & \multirow{2}{*}{$\begin{array}{l}\text { Mulheres com } \\
25-59 \text { anos } \\
\text { Exame do } \\
\text { Papanicolau } \\
\text { realizado há } 3 \text { anos } \\
\text { ou menos }\end{array}$} & \multirow{2}{*}{$\begin{array}{l}\text { Mulheres com } \\
50-69 \text { anos } \\
\text { Mamografia } \\
\text { realizada há } 2 \text { anos } \\
\text { ou menos }\end{array}$} & \multirow{2}{*}{$\begin{array}{c}\text { Homens e } \\
\text { mulheres com } 60 \\
\text { anos ou mais } \\
\text { Vacinação contra a } \\
\text { gripe nos últimos } \\
12 \text { meses }\end{array}$} \\
\hline & $\begin{array}{l}\text { Pressão arterial } \\
\text { aferida há } 2 \text { anos } \\
\text { ou menos }\end{array}$ & $\begin{array}{l}\text { Colesterol aferido } \\
\text { há } 5 \text { anos ou } \\
\text { menos }\end{array}$ & & & \\
\hline & $\%$ (IC95\%) & $\%$ (IC95\%) & $\%$ (IC95\%) & $\%$ (IC95\%) & $\%$ (IC95\%) \\
\hline Grupo I: UBS "tradicional" & $89,9(87,4-92,3)$ & $94,1(92,2-96,0)$ & $85,3(80,9-89,7)$ & $79,8(71,3-88,6)$ & $81,8(75,1-88,4)$ \\
\hline Grupo II: plano privado de saúde & $96,2(95,4-97,1) *$ & $97,2(96,5-97,9)$ * & $91,1(89,1-93,0)$ * & $85,0(81,2-88,8)$ & $82,6(79,0-86,3)$ \\
\hline $\begin{array}{l}\text { Grupo III: coberto pela ESF e } \\
\text { usuário não regular }\end{array}$ & $90,3(88,0-92,7)$ & $89,7(87,2-92,2)$ * & $83,2(78,3-88,2)$ & $81,4(72,7-90,0)$ & $78,9(71,5-86,2)$ \\
\hline $\begin{array}{l}\text { Grupo IV: cobertos pela ESF e } \\
\text { provável usuário regular }\end{array}$ & $90,4(88,4-92,5)$ & $95,0(93,7-96,4)$ & $88,6(85,7-91,4)$ & $80,5(74,0-86,9)$ & $84,8(79,8-89,7)$ \\
\hline
\end{tabular}

ESF: Estratégia Saúde da Família; IC95\%: intervalo de 95\% de confiança; UBS: unidade básica de saúde.

Nota: porcentagem ajustada por fatores predisponentes, de necessidade e facilitadores do uso de serviços de saúde.

${ }^{*} p<0,05$ para diferenças em relação ao grupo I.

dos exames de rastreamento e da vacinação contra a gripe apresentaram variações pouco importantes entre as diferentes fontes de atenção em saúde. Esses resultados foram independentes de fatores predisponentes (características demográ-

ficas) de necessidade (condições de saúde) e facilitadores (condições socioeconômicas) do uso de serviços de saúde. 
Na presente análise, foram considerados quatro indicadores de uso de serviços de saúde, três dos quais estão inter-relacionados: existência de fonte regular de serviços de saúde (uma medida da longitudinalidade), procura recente por atenção à saúde e realização de uma ou mais consultas médicas no último ano. A necessidade de pelo menos uma consulta anual é fundamentada por evidências de que o adulto sem diagnóstico de alguma doença deve ter pelo menos quarenta minutos de atenção médica por ano, acrescidos de vinte minutos para cada condição crônica que ele apresente 27 . Evidências também indicam que o alcance dessa meta está fortemente associado à existência de uma fonte regular de serviços de saúde 28 . Nossos resultados mostram que a prevalência de fonte regular de serviços foi baixa, tanto no sistema público quanto no privado, sobretudo entre residentes em área coberta pela USB "tradicional" e residentes em área coberta pela ESF, mas não usuários regulares da mesma. Isso aponta para problemas organizacionais dos serviços de saúde no sentido de estreitar o vínculo e proporcionar o acompanhamento dos pacientes.

Por outro lado, a ocorrência de hospitalizações não variou significativamente entre as diferentes fontes de atenção, apontando para a existência de equidade no acesso às instituições. Essa observação é importante porque as hospitalizações geralmente indicam uma necessidade grave e urgente de cuidados médicos e de tecnologias especializadas, ao passo que as consultas médicas refletem não apenas necessidades, mas também outros aspectos da demanda individual.

A percepção dos usuários acerca do sistema de saúde e da atenção prestada quando precisam de serviços de saúde é fundamental para avaliar a sua qualidade. Muitas vezes, o desempenho dos serviços de saúde avaliado por meio de indicadores de processo pode mostrar bons resultados, mas tais indicadores não captam as expectativas e as necessidades daqueles que usam os serviços 29. Os resultados do presente trabalho mostram que cerca de $60 \%$ dos usuários do sistema público informaram ter alguma dificuldade para conseguir consultas médicas. A existência de filas é a principal barreira apontada. Esses resultados contrastam de forma marcante com os obtidos entre os afiliados a plano privado de saúde. Entre estes, o relato de dificuldades para a obtenção de consultas e da existência de filas foram duas a três vezes menos frequentes. Coerentemente, a proporção dos que informaram obter consultas num prazo de 24 horas foi significativamente mais alta entre afiliados a plano privado de saúde, em relação aos demais grupos.
A queixa para obtenção medicamentos de uso regular foi mais alta entre residentes na área coberta pela USB "tradicional” e mais baixa entre residentes em áreas cobertas pela ESF (tanto entre prováveis usuários regulares quanto entre não usuários regulares), assim como entre afiliados a plano privado de saúde. Em certa medida, esses resultados são consonantes com um estudo baseado na PNAD 2008, mostrando que o acesso gratuito a medicamentos de uso regular é maior entre usuários da ESF do que entre cobertos pelos demais modelos de atenção ${ }^{30}$. Essa iniquidade não se justifica, uma vez que o governo brasileiro tem feito esforços bem sucedidos para aumentar o acesso gratuito a medicamentos essenciais 31,32

A percepção de que a consulta médica dura menos que o necessário predominou amplamente em todos os grupos analisados, revelando insatisfação tanto entre os afiliados a plano privado de saúde quanto entre os que dependem do SUS para cuidados com a saúde. A adequação do tempo da consulta é importante para que o indivíduo tenha as suas necessidades atendidas, entenda as recomendações médicas e estabeleça vínculo com o profissional de saúde. Os dois últimos são importantes determinantes da adesão ao aconselhamento sobre comportamentos saudáveis e uso apropriado dos medicamentos prescritos 33 .

Deve-se salientar que a recomendação dos médicos ou serviços que costumam procurar (um indicador geral de satisfação) variou amplamente entre os diferentes modelos de atenção. A proporção de satisfeitos foi maior entre prováveis usuários regulares da ESF e entre afiliados a plano privado de saúde. A proporção de insatisfeitos foi mais alta entre residentes em área coberta pela UBS "tradicional", assim como entre residentes em área coberta pela ESF, mas não usuários regulares dela.

Outro indicador de qualidade da atenção primaria é a cobertura dos exames de rastreamento. Existem grupos de trabalho internacionalmente respeitados, que produzem recomendações baseadas nas evidências científicas disponíveis, estabelecendo quais exames devem ser realizados, a sua periodicidade e as faixas etárias alvo. O Ministério da Saúde adota as recomendações da U.S. Preventive Services Task Force (USPSTF), com pequenas adaptações 20. Nos Estados Unidos, são estabelecidas metas para a cobertura populacional dos exames de rastreamento. Para 2020, as metas a serem alcançadas são: aferição da pressão arterial a cada três anos em $94 \%$ da população com idade igual ou superior a 18 anos; dosagem do colesterol a intervalo de pelo menos cinco anos em $82 \%$ da população com 
idade igual ou superior a 18 anos; realização da mamografia a intervalo de pelo menos dois anos em $81 \%$ das mulheres entre 50 e 74 anos; realização do Papanicolau a intervalo de pelo menos três anos em 93\% das mulheres com 21 a 65 anos 22. Os resultados do presente trabalho mostram que, à exceção do exame do Papanicolau, em todos os grupos analisados, a cobertura dos exames de rastreamento estava próxima (ou mesmo havia superado) as metas acima mencionadas. Com referência ao exame do Papanicolau, a sua cobertura alcançou as metas norteamericanas entre as afiliadas a plano privado de saúde. Nos demais grupos, ela foi superior a $80 \%$. A Organização Mundial da Saúde (OMS) avalia que, com uma cobertura populacional mínima de $80 \%$ e a garantia de diagnóstico e tratamento adequados, é possível reduzir em média $60 \%$ a $90 \%$ da incidência de câncer invasivo de colo de útero na população 20 . A vacinação contra a gripe em idosos foi semelhante nos diferentes grupos, com proporção de cobertura em torno de $80 \%$. Em comparação a 2003, quando foi realizado o primeiro inquérito de saúde de Belo Horizonte, a cobertura da vacinação contra a gripe aumentou cerca de $20 \% 33$.

Este trabalho tem vantagens e limitações. As principais vantagens são a grande base populacional investigada, a sua representatividade, a variedade de indicadores utilizados como variáveis dependentes, assim como a inclusão na análise multivariada de fatores predisponentes, de necessidade e facilitadores do uso de serviços de saúde. Outra vantagem foi a existência, no Município de Belo Horizonte, de critérios claramente definidos para as áreas de implantação da ESF. Isso possibilitou a localização precisa - por meio de georreferenciamento - dos domicílios situados em áreas cobertas e não cobertas por essa estratégia. Por outro lado, não foi possível identificar os indivíduos que de fato eram usuários e não usuários da ESF, uma vez que $27 \%$ dos participantes do estudo não sabiam informar 17. Diante dessa limitação, optou-se por criar dois grupos de residentes em área coberta pela ESF, constituídos por prováveis usuários regulares e não regulares. Para a construção desses grupos, assumiu-se a premissa de que aqueles que não sabiam informar eram improváveis usuários re- gulares da ESF. Outra premissa assumida neste trabalho foi a de que os afiliados a plano privado de saúde não usam os serviços públicos para atendimento ambulatorial. Tais premissas parecem plausíveis, mas não podem ser confirmadas por fonte independente. Finalmente, seria desejável que os quatro grupos comparados fossem semelhantes em todas as suas características, exceto a fonte de atenção à saúde. Essa situação ideal, contudo, não é possível, uma vez que a situação socioeconômica é determinante da adesão a plano privado de saúde e da cobertura pela ESF. Para contornar esse problema, optou-se por ajustar nossas estimativas pelo nível de escolaridade e pelo escore de bens domiciliares (dois robustos indicadores de condições socioeconômicas), após verificar que não havia colinearidade entre essas duas variáveis. Entretanto, não se pode descartar a possibilidade de que outros fatores não mensurados possam ter afetado nossos resultados. De qualquer forma, as variações observadas entre as várias fontes de serviços de saúde foram marcantes, sendo pouco provável que elas sejam resultantes de vieses.

Os resultados do presente trabalho mostram heterogeneidade no perfil de utilização e na qualidade dos serviços oferecidos pelas diferentes fontes de atenção em saúde. Essas diferenças são consequência tanto das desigualdades socioeconômicas (como no caso dos planos privados $v s$. sistema público), quanto de diferenças na organização da atenção no âmbito do SUS (ESF vs. UBS “tradicional”). Cabe salientar que a maioria dos indicadores de uso de serviços de saúde apresentou melhor performance entre prováveis usuários regulares da ESF, em comparação aos residentes em área coberta pela UBS "tradicional", e que somente um indicador de qualidade na percepção dos usuários - existência de filas - apresentou melhor performance entre os últimos em comparação aos primeiros. Além disso, a recomendação para outra pessoa dos serviços de saúde utilizados foi mais frequente entre prováveis usuários regulares da ESF e afiliados a plano privado de saúde, em comparação à UBS "tradicional". Esses resultados reforçam a importância dessa estratégia de atenção à saúde em um município de grande porte, como Belo Horizonte. 


\section{Resumen}

Se compararon indicadores de uso y calidad de los servicios de salud en una muestra probabilística de adultos $(N=7.534)$, cubiertos por un plan privado de salud, Estrategia Salud de la Familia (ESF) y la unidad básica de salud (UBS) "tradicional" en el municipio de Belo Horizonte, Minas Gerais, Brasil. Tras ajustes por factores demográficos, condiciones de salud y situación socioeconómica, los indicadores de uso (longitudinalidad, búsqueda de atención y consultas médicas) presentaron mejores resultados entre usuarios regulares de la ESF y afiliados a un plan privado de salud, en comparación con quienes estaban cubiertos por la UBS. Las hospitalizaciones, los exámenes de rastreo y la vacunación contra la gripe variaron poco entre esos grupos. Los indicadores de calidad (dificultades para obtener consultas, existencia de colas, reclamaciones para la obtención de medicamentos y recibir consultas en 24 horas) fueron mejores entre los afiliados a un plan de salud privado. La recomendación de los servicios de salud utilizados a otra persona fue más frecuente entre usuarios regulares de la ESF (61,9\%) y los afiliados a un plan privado de salud (55,6\%), en comparación con la UBS $(45,4 \%)$.

Accesibilidad a los Servicios de Salud; Indicadores de Calidad de la Atención de Salud; Planes de Salud de Prepago

\section{Colaboradores}

M. F. Lima-Costa e J. Macinko participaram da concepção do projeto, análise dos dados, redação do artigo, revisão crítica do trabalho e aprovaram a versão final M. A. Turci colaborou na concepção do projeto, revisão crítica do trabalho e aprovou a versão final.

\section{Agradecimentos}

O segundo Inquérito de Saúde da Região Metropolitana de Belo Horizonte foi financiado pelo Departamento de Atenção Básica da Secretaria de Assistência à Saúde do Ministério da Saúde. Os autores agradecem a Breno Dayrell Bretas Alvarenga Bastos, bioestatístico bolsista da Fundação Oswaldo Cruz em Minas Gerais, pela cuidadosa preparação do banco de dados para análise, e a Walter Luiz Batista Ferraz, da Secretaria Municipal de Saúde de Belo Horizonte, pelo georreferenciamento dos domicílios participantes da pesquisa.

\section{Referências}

1. Starfield B. Primary care: balancing health needs, services and technology. New York: Oxford University Press; 1998.

2. Paim J, Travassos C, Almeida C, Bahia L, Macinko J. The Brazilian health system: history, advances, and challenges. Lancet 2011; 377:1778-97.

3. Portela GZ, Ribeiro JM. A sustentabilidade econômico-financeira da Estratégia da Saúde da Família em municípios de grande porte. Ciênc Saúde Coletiva $2011 ; 16: 1719-32$.

4. Macinko J, Souza MFM, Guanais FC, Simões CCS. Going to scale with community-based primary care: an analysis of the family health program and infant mortality in Brazil, 1999-2004. Soc Sci Med 2007; 65:2070-80.

5. Aquino R, Oliveira NF, Barreto ML. Impact of the family health program on infant mortality in Brazilian municipalities. Am J Public Health 2009; 99:87-93.

6. Macinko J, Dourado I, Aquino R, Bonolo PF, LimaCosta MF, Medina MG, et al. Major expansion of primary care in Brazil linked to decline of unnecessary hospitalization. Health Aff (Millwood) 2010; 29:2149-60.
7. Macinko J, Oliveira VB, Turci MA, Guanais FC, Bonolo PF, Lima-Costa MF. The influence of primary care and hospital supply on ambulatory-care sensitive hospitalizations among adults in Brazil, 1999-2007. Am J Public Health 2011; 101:1963-70.

8. Dourado I, Oliveira VB, Aquino R, Bonolo P, LimaCosta MF, Medina MG, et al. Trends in primary health care-sensitive conditions in Brazil: the role of the Family Health Program (Project ICSAP-Brazil). Med Care 2011; 49:577-84.

9. Macinko J, Lima-Costa MF. Access to, use of and satisfaction with health services among adults enrolled in Brazil's Family Health Strategy: evidence from the 2008 National Household Survey. Trop Med Int Health 2011; 17:36-42.

10. Rodrigues MAP, Facchini LA, Piccini RX, Tomasi E, Thumé E, Silveira DS, et al. Uso de serviços ambulatoriais por idosos nas regiões Sul e Nordeste do Brasil. Cad Saúde Pública 2008; 24:2267-78.

11. Paniz VMV, Fassa AG, Facchini LA, Bertoldi AD, Piccini RX, Tomais E, et al. Acesso a medicamentos de uso contínuo em adultos e idosos nas regiões Sul e Nordeste do Brasil. Cad Saúde Pública 2008; 24:267-80 
12. Viana ALd'A, Rocha JSY, Elias PE, Ibañez N, Bouquat A. Atenção básica e dinâmica urbana em grandes municípios paulistas. Cad Saúde Pública 2008; 24 Suppl 1:S79-90.

13. Elias PE, Ferreira CW, Alves MCG, Cohn A, Kishima V, Escrivão Junior A, et al. Atenção básica em saúde: comparação entre PSF e UBS por estrato de exclusão social no município de São Paulo. Ciênc Saúde Coletiva 2006; 11:633-41.

14. Mendonça CS, Harzheim E, Duncan BB, Nunes LN, Leiyh W. Trends in hospitalizations for primary care sensitive conditions following the implementation of Family Health Teams in Belo Horizonte, Brazil. Health Policy Plan 2012; 27:348-55.

15. Instituto Brasileiro de Geografia e Estatística. In dicadores sociais municipais: uma análise dos resultados do universo do Censo Demográfico 2010. Rio de Janeiro: Instituto Brasileiro de Geografia e Estatística; 2011. (Estudos e Pesquisas. Informação Demográfica e Socioeconômica, 28).

16. Turci MA, Lima-Costa MF, Proietti FA, Cesar CC, Macinko J. Intraurban differences in the use of ambulatory health services in a large Brazilian city. J Urban Health 2010; 87:994-1006.

17. Lima-Costa MF, Turci M, Macinko J. Saúde dos adultos em Belo Horizonte. Belo Horizonte: Núcleo de Estudos em Saúde Pública e Envelhecimento, Fundação Oswaldo Cruz/Universidade Federal de Minas Gerais; 2012.

18. Turci MA, organizadora. Avanços e desafios na organização da atenção de saúde em Belo Horizonte. Belo Horizonte: Secretaria Municipal de Saúde de Belo Horizonte; 2008.

19. Centro de Estatística e Informações, Fundação João Pinheiro. Pesquisa de emprego e desemprego na Região Metropolitana de Belo Horizonte PED/ RMBH: boletim anual 2010. Belo Horizonte: Fundação João Pinheiro; 2011.

20. Departamento de Atenção Básica, Secretaria de Atenção à Saúde, Ministério da Saúde. Rastreamento. Brasília: Ministério da Saúde; 2010. (Série A. Normas e Manuais Técnicos). (Cadernos de Atenção Primária, 29).

21. Instituto Nacional de Câncer. Diretrizes brasileiras para o rastreamento do câncer do colo do útero. Rio de Janeiro: Instituto Nacional de Câncer; 2011.
22. U.S. Department of Health and Human Services Healthy people 2020. http://www.healthypeople. gov/2020/topicsobjectives2020/pdfs/HP2020ob jectives.pdf (acessado em 23/Mai/2010).

23. Andersen R, Newman JF. Societal and individual determinants of medical care utilization in the United States. Milbank Mem Fund Q Health Soc 1973; 51:95-124.

24. Long JS, Freese J. Regression model for categorical dependent variables using Stata. 2nd Ed. College Station: Stata Press; 2006.

25. Cummings $P$. The relative merits of risk ratios and odds ratios. Arch Pediatr Adolesc Med 2009; 163:438-45.

26. Ismail K. Unravelling factor analysis. Evid Based Ment Health 2008; 11:99-102.

27. Yarnall KS, Pollak KI, Østbye T, Krause KM, Michener JL. Primary care: is there enough time for prevention? Am J Public Health 2003; 93:635-41.

28. Ettner SL. The timing of preventive services for women and children: the effect of having a usual source of care. Am J Public Health 1996; 86:1748-54.

29. Macinko J, Lima-Costa MF. Access, use and satisfaction with health services in Brazil's Family Health Strategy. Trop Med Int Health 2012; 17:36-42.

30. Roter D. The enduring and evolving nature of the patient-physician relationship. Patient Educ Couns 2000; 39:5-15.

31. Secretaria de Políticas de Saúde, Ministério da Saúde. Portaria no 3.916, de 30 de outubro de 1998, dispõe sobre a aprovação da Política Nacional de Medicamentos. Diário Oficial da União 1998; 31 out.

32. Santos-Pinto CB, Costa NR, Osório-de-Castro CG. Quem acessa o Programa Farmácia Popular do Brasil? Aspectos do fornecimento público de medicamentos. Ciênc Saúde Coletiva 2011; 16:2963-73.

33. Lima-Costa MF. Fatores associados à vacinação contra gripe em idosos na região metropolitana de Belo Horizonte. Rev Saúde Pública 2008; 42:100-7.

Recebido em 13/Mar/2012

Versão final reapresentada em 16/Jun/2012

Aprovado em 01/Abr/2013 